\title{
Inverse problem for Dirac systems with locally square-summable potentials and rectangular Weyl functions
}

\author{
Alexander Sakhnovich
}

\begin{abstract}
Inverse problem for Dirac systems with locally square summable potentials and rectangular Weyl functions is solved. For that purpose we use a new result on the linear similarity between operators from a subclass of triangular integral operators and the operator of integration.
\end{abstract}

Mathematics Subject Classification (2010). Primary: 34A55, 34B20, 34L40; Secondary: 47A48, 47G10.

Keywords. Dirac system, Weyl function, inverse problem, rectangular potential, similarity transformation.

\section{Introduction}

We consider the self-adjoint Dirac (more precisely, Dirac-type) system

$$
\frac{d}{d x} y(x, z)=\mathrm{i}(z j+j V(x)) y(x, z) \quad(x \geq 0),
$$

where

$$
j=\left[\begin{array}{cc}
I_{m_{1}} & 0 \\
0 & -I_{m_{2}}
\end{array}\right], \quad V=\left[\begin{array}{cc}
0 & v \\
v^{*} & 0
\end{array}\right], \quad m_{1}+m_{2}=: m,
$$

$I_{m_{k}}$ is the $m_{k} \times m_{k}$ identity matrix and $v(x)$ is an $m_{1} \times m_{2}$ matrix function. We assume that $v$ is measurable and, moreover, locally square-summable, that is, square-summable on the finite intervals $[0, l]$. Here we say that a matrix function is summable (square-summable) if its entries are summable (square-summable). 
Dirac (Dirac-type) system is a classical object of analysis. Its Weyl and spectral theories were actively studied in the second half of the 20-th century, the first solution of the inverse spectral problem being given (for the case of the scalar $v$ and without proof) by M.G. Krein in the seminal paper [11]. For more recent publications on Dirac systems see, for instance, $[1,2,3,5,6,8,12,13,16,17]$ and references therein. Dirac system is of independent interest and it is also important as an auxiliary system for many integrable nonlinear equations. Moreover, it is related to the famous Schrödinger equation (see, e.g., [4]). Many recent publications are dedicated to the development of the Weyl and spectral theories of Dirac system under weaker summability conditions. Here, we solve the inverse problem under the condition of the local square-summability of $v$. We deal with the case where the potential $v$ and the corresponding Weyl function are rectangular (not necessarily square) matrix functions, which is essential for some applications to matrix and multicomponent integrable equations.

Before stating our main result, we formulate several results from [6, 17] on direct problems. The notation $u(x, z)$ stands for the fundamental solution of (1.1) normalized by the condition

$$
u(0, z)=I_{m} .
$$

Later we shall need notations of the block rows of $u(x, 0)$ :

$$
\beta(x)=\left[\begin{array}{ll}
I_{m_{1}} & 0
\end{array}\right] u(x, 0), \quad \gamma(x)=\left[\begin{array}{ll}
0 & I_{m_{2}}
\end{array}\right] u(x, 0) .
$$

Definition 1.1. A Weyl-Titchmarsh (or simply Weyl) function of Dirac system (1.1) on $[0, \infty)$, where the potential $v$ is locally summable, is a holomorphic $m_{2} \times m_{1}$ matrix function $\varphi$ which satisfies the inequality

$$
\int_{0}^{\infty}\left[\begin{array}{ll}
I_{m_{1}} & \varphi(z)^{*}
\end{array}\right] u(x, z)^{*} u(x, z)\left[\begin{array}{c}
I_{m_{1}} \\
\varphi(z)
\end{array}\right] d x<\infty, \quad z \in \mathbb{C}_{+} .
$$

Here $\mathbb{C}$ denotes the complex plane and $\mathbb{C}_{+}$stands for the open upper half-plane. In order to study Weyl functions, we introduce the class of nonsingular $m \times m_{1}$ matrix functions $\mathcal{P}(z)$ with property- $j$. Namely, the matrix functions $\mathcal{P}(z)$ are meromorphic in $\mathbb{C}_{+}$and satisfy (excluding, possibly, a discrete set of points) the following relations

$$
\mathcal{P}(z)^{*} \mathcal{P}(z)>0, \quad \mathcal{P}(z)^{*} j \mathcal{P}(z) \geq 0 \quad\left(z \in \mathbb{C}_{+}\right) .
$$

Relations (1.6) imply

$$
\operatorname{det}\left(\left[\begin{array}{ll}
I_{m_{1}} & 0
\end{array}\right] u(x, z)^{-1} \mathcal{P}(z)\right) \neq 0 .
$$


Definition 1.2. The set $\mathcal{N}(x, z)$ of Möbius transformations is the set of values at $x, z$ of matrix functions

$$
\varphi(x, z, \mathcal{P})=\left[\begin{array}{ll}
0 & I_{m_{2}}
\end{array}\right] u(x, z)^{-1} \mathcal{P}(z)\left(\left[\begin{array}{ll}
I_{m_{1}} & 0
\end{array}\right] u(x, z)^{-1} \mathcal{P}(z)\right)^{-1},
$$

where $\mathcal{P}(z)$ are nonsingular matrix functions with property- $j$.

As usual, the sets $\mathcal{N}(x, z)$ are embedded, that is,

$$
\mathcal{N}\left(x_{1}, z\right) \subset \mathcal{N}\left(x_{2}, z\right) \text { for } x_{1}>x_{2} .
$$

Moreover, the following proposition holds.

Proposition 1.3. [17, Subsection 2.2.1] Let Dirac system (1.1) be given on $[0, \infty)$ and let its potential $v$ be locally summable. Then there is a unique matrix function $\varphi(z)$ in $\mathbb{C}_{+}$such that

$$
\varphi(z)=\bigcap_{x<\infty} \mathcal{N}(x, z)
$$

This function is analytic and non-expansive. Moreover, this function is the unique Weyl function of system (1.1).

If $v$ is locally square-summable, we may recover it from the Weyl function.

Theorem 1.4. Let Dirac system (1.1) be given on $[0, \infty)$, let its potential $v$ be locally square-summable and let $\varphi$ be the Weyl function of this system. Then $v$ is uniquely recovered from $\varphi$.

The procedure to recover $v$ from $\varphi$ is based on the study of the operator

$$
K=\mathrm{i} \int_{0}^{x} \gamma(x) j \gamma(t)^{*} \cdot d t, \quad K \in B\left(L_{m_{2}}^{2}(0, l)\right),
$$

where $\gamma$ is the lower block row of $u(x, 0)$ (see $(1.4)), L_{r}^{2}(0, l)$ is the class of square summable vector functions on $(0, l)$ with values in $\mathbb{C}^{r}$ and scalar product $(f, g)=$ $\int_{0}^{l} g(x)^{*} f(x) d x$, and $B(H)$ denotes the class of bounded linear operators, which map the space $H$ into $H$. Using a new version of the similarity result for $K$, we modify for the case of the locally square-summable potentials $v$ the procedure to solve inverse problem, which was developed in $[15,16,17]$.

Further, $F^{\prime}$ stands for the derivative of $F$, "const" means a constant function or vector-function, $I_{r}$ is the $r \times r$ identity matrix, $I$ is an identity operator, $B\left(H_{1}, H_{2}\right)$ denotes the class of bounded linear operators, which map the Hilbert space $H_{1}$ into the Hilbert space $\mathrm{H}_{2}$. When speaking about fundamental solutions, we assume that they are normalized by $I_{m}$ at $x=0$. 


\section{Similarity result}

We consider conditions of similarity of the two operators acting in $L_{r}^{2}(0, \mathbf{T})$, namely,

$$
K:=F(x) \int_{0}^{x} G(t) \cdot d t, \quad A:=\int_{0}^{x} \cdot d t,
$$

where $F$ and $G$ are differentiable $r \times p$ and $p \times r$, respectively, matrix functions.

Proposition 2.1. Let $F$ and $G$ be differentiable and satisfy the identity

$$
F(x) G(x) \equiv I_{r}, \quad 0 \leq x \leq \mathbf{T},
$$

and assume that the entries of $F^{\prime}$ and $G^{\prime}$ belong to $L^{2}(0, \mathbf{T})$.

Then the operator $K$ defined by (2.1) is similar to the operator of integration $A$. More precisely, $K=E A E^{-1}$ where $E \in B\left(L_{r}^{2}(0, \mathbf{T})\right)$ is a lower triangular operator of the form

$$
E=\rho(x)\left(I+\int_{0}^{x} N(x, t) \cdot d t\right), \quad \frac{d}{d x} \rho=F^{\prime} G \rho, \quad \rho(0)=I_{r},
$$

and the matrix functions $\rho, \rho^{-1}$ and $N$ are measurable and uniformly bounded. Moreover, the operators $E^{ \pm 1}$ map differentiable functions with a square-summable derivative into differentiable functions with a square-summable derivative.

The case of operators $K$ of the form (2.1), where $F$ and $G$ have bounded derivatives, is a particular case of operators, the similarity of which to $A$ was proved in an important paper [18]. Later on, the proof from [18] was modified for the case of operators $K$ such that $F$ and $G$ have continuous derivatives (and $E^{ \pm 1}$ map functions with continuous derivatives into functions with continuous derivatives) [2]. Here, we modify further the proofs from $[2,18]$ for the case of the less smooth functions $F$ and $G$. The proof of Proposition 2.1 above requires some preparations.

We note that, according to the general theory of semi-separable integral operators, which is also easily checked directly, the inverse of operator $I-z K$ is given by

$$
\left((I-z K)^{-1} f\right)(x)=f(x)+\int_{0}^{x} Q(x, t, z) f(t) d t,
$$

where

$$
\begin{aligned}
Q(x, t, z) & =z F(x) u_{1}(x, z) u_{1}(t, z)^{-1} G(t), & & 0 \leq t \leq x \leq \mathbf{T} \\
\frac{d}{d x} u_{1}(x, z) & =z G(x) F(x) u_{1}(x, z), & & 0 \leq x \leq \mathbf{T} \\
u_{1}(0, z) & =I_{r} . & &
\end{aligned}
$$


Introduce also the $p \times p$ matrix function $\tilde{u}_{1}(x)$ defined by

$$
\frac{d}{d x} \tilde{u}_{1}(x)=-G(x) F^{\prime}(x) \tilde{u}_{1}(x), \quad 0 \leq x \leq \mathbf{T}, \tilde{u}_{1}(0)=I_{p} .
$$

We are now ready to prove the first lemma.

Lemma 2.2. Let $F$ and $G$ be absolutely continuous and assume that the identity (2.2) holds. Introduce the $r \times r$ matrix functions $h$ and $\rho$ by

$$
h(x):=F(x) G(0) ; \quad \frac{d}{d x} \rho=F^{\prime} G \rho, \quad \rho(0)=I_{r} .
$$

Put

$$
g(x, z)=\rho(x)^{-1}\left((I-z K)^{-1} h\right)(x), \quad 0 \leq x \leq \mathbf{T},
$$

where $(I-z K)^{-1}$ is applied to h columnwise. Then $g$ satisfies the following integro-differential equation

$$
\frac{d}{d x} g(x, z)-\mu(x) \int_{0}^{x} v(t) g(t, z) d t-z g(x, z)=0, \quad g(0, z)=I_{r},
$$

where $\mu$ and $v$ are the summable functions on $[0, \mathbf{T}]$ given by

$$
\begin{array}{rlrl}
\mu(x) & :=\rho(x)^{-1} F^{\prime}(x) \tilde{u}_{1}(x), & & 0 \leq x \leq \mathbf{T} ; \\
\nu(t):=-\tilde{u}_{1}(t)^{-1}\left(G(t) F^{\prime}(t) G(t)+G^{\prime}(t)\right) \rho(t), & 0 \leq t \leq \mathbf{T} .
\end{array}
$$

Proof. Put $\tilde{g}(x, z)=\rho(x) g(x, z)$. Using (2.4)-(2.7), (2.10), and the definition of the matrix function $h$, we present $\tilde{g}$ in the form

$$
\begin{aligned}
\tilde{g}(x, z) & =F(x) G(0)+z F(x) u_{1}(x, z) \int_{0}^{x} u_{1}(t, z)^{-1} G(t) F(t) G(0) d t \\
& =F(x) G(0)-F(x) u_{1}(x, z) \int_{0}^{x} \frac{d}{d t}\left(u_{1}(t, z)^{-1} G(0)\right) d t \\
& =F(x) G(0)-F(x) u_{1}(x, z)\left(u_{1}(x, z)^{-1}-I_{r}\right) G(0) \\
& =F(x) u_{1}(x, z) G(0) .
\end{aligned}
$$

It follows that

$$
g(x, z)=\rho(x)^{-1} F(x) u_{1}(x, z) G(0) .
$$

Clearly $g$ is differentiable and

$$
\begin{aligned}
\frac{d}{d x} g(x, z) & =\rho(x)^{-1} \tilde{g}_{x}(x, z)-\rho(x)^{-1} \rho^{\prime}(x) \rho(x)^{-1} \tilde{g}(x, z) \\
& =\rho(x)^{-1}\left\{z F(x) G(x) F(x)+F^{\prime}(x)-F^{\prime}(x) G(x) F(x)\right\} u_{1}(x, z) G(0) \\
& =z g(x, z)+\rho(x)^{-1} F^{\prime}(x)\left(I_{p}-G(x) F(x)\right) u_{1}(x, z) G(0) .
\end{aligned}
$$


Here we took into account the identity (2.2). From (2.8) we see that

$$
\frac{d}{d t} \tilde{u}_{1}(t)^{-1}=-\tilde{u}_{1}(t)^{-1}\left(\frac{d}{d t} \tilde{u}_{1}(t)\right) \tilde{u}_{1}(t)^{-1}=\tilde{u}_{1}(t)^{-1} G(t) F^{\prime}(t)
$$

Hence

$$
\begin{aligned}
\frac{d}{d t}\left(\tilde { u } _ { 1 } ( t ) ^ { - 1 } \left(I_{p}\right.\right. & \left.-G(t) F(t)) u_{1}(t, z)\right) \\
=\tilde{u}_{1}(t)^{-1} G(t) F^{\prime}(t)\left(I_{p}-G(t) F(t)\right) u_{1}(t, z) & \\
& +\tilde{u}_{1}(t)^{-1}\left(-G^{\prime}(t) F(t)-G(t) F^{\prime}(t)\right) u_{1}(t, z) \\
& +z \tilde{u}_{1}(t)^{-1}\left(I_{p}-G(t) F(t)\right) G(t) F(t) u_{1}(t, z) .
\end{aligned}
$$

Since, in view of condition (2.2), we have $\left(I_{p}-G(t) F(t)\right) G(t)=0$, we obtain

$$
\begin{aligned}
& \frac{d}{d t}\left(\tilde{u}_{1}(t)^{-1}\left(I_{p}-G(t) F(t)\right) u_{1}(t, z)\right) \\
& =\tilde{u}_{1}(t)^{-1}\left(G(t) F^{\prime}(t)-G(t) F^{\prime}(t) G(t) F(t)\right. \\
& \left.-G^{\prime}(t) F(t)-G(t) F^{\prime}(t)\right) u_{1}(t, z) \\
& =-\tilde{u}_{1}(t)^{-1}\left(G(t) F^{\prime}(t) G(t)+G^{\prime}(t)\right) F(t) u_{1}(t, z) \text {. }
\end{aligned}
$$

Using the definition of $v$ in (2.13) and the identity (2.15), we derive

$$
\frac{d}{d t}\left(\tilde{u}_{1}(t)^{-1}\left(I_{p}-G(t) F(t)\right) u_{1}(t, z)\right) G(0)=v(t) g(t, z) .
$$

Recall that $\left(I_{p}-G(t) F(t)\right) G(t)=0$ and so $\left(I_{p}-G(0) F(0)\right) G(0)=0$, in particular. Hence, from (2.17) it follows that

$$
\begin{array}{rl}
\int_{0}^{x} & v(t) g(t, z) d t \\
& =\tilde{u}_{1}(x)^{-1}\left(I_{p}-G(x) F(x)\right) u_{1}(x, z) G(0)-\left(I_{p}-G(0) F(0)\right) G(0) \\
& =\tilde{u}_{1}(x)^{-1}\left(I_{p}-G(x) F(x)\right) u_{1}(x, z) G(0) .
\end{array}
$$

But then, using (2.16) and the definition of $\mu$ in (2.12), we arrive at the identity (2.11).

The lemma below provides an integral representation of the solution of (2.11). 
Lemma 2.3. Let $\mu(x)$ and $v(x)$ be $r \times p$ and $p \times r$, respectively, matrix functions, such that their entries belong to $L^{2}(0, \mathbf{T})$. Then the integro-differential equation

$$
\begin{aligned}
& \frac{d}{d x} g(x, z)-\int_{0}^{x} \varkappa(x, t) g(t, z) d t-z g(x, z)=0, \quad g(0, z)=I_{r}, \\
& \varkappa(x, t):=\mu(x) v(t)
\end{aligned}
$$

has a unique solution $g(\cdot, z) \in L_{r}^{2}(0, \mathbf{T})$, and this solution has the form

$$
g(x, z)=e^{z x} I_{r}+\int_{0}^{x} e^{z t} N(x, t) d t, \quad 0 \leq x \leq \mathbf{T},
$$

where $N(x, t)$ is bounded on $0 \leq t \leq x \leq \mathbf{T}$.

Proof. We set

$$
\begin{aligned}
& \varkappa_{1}(x, t)=\int_{x-t}^{x} \varkappa(\xi, \xi+t-x) d \xi, \quad 0 \leq t \leq x \leq \mathbf{T}, \\
& \varkappa_{k+1}(x, t)=\int_{x-t}^{x} \int_{y+t-x}^{y} \varkappa(y, s) \varkappa_{k}(s, y+t-x) d s d y .
\end{aligned}
$$

It is easily proved by induction that

$$
\left\|\varkappa_{k}(x, t)\right\| \leq C_{0} C_{1}^{k-1} \frac{x^{k-1}}{(k-1) !}, \quad 0 \leq t \leq x \leq \mathbf{T}, k \geq 1
$$

for some $C_{0}, C_{1}>0$. Thus, we can introduce a bounded matrix function

$$
N(x, t)=\sum_{k=1}^{\infty} \varkappa_{k}(x, t), \quad 0 \leq t \leq x \leq \mathbf{T} .
$$

Putting

$$
\mathfrak{G}_{0}(x, t)=\varkappa(x, t) ; \quad \mathfrak{G}_{k}(x, t)=\int_{t}^{x} \varkappa(x, s) \varkappa_{k}(s, t) d s, \quad k>0,
$$

and using (2.22), (2.23), and (2.26), we easily derive

$$
\begin{aligned}
\int_{0}^{x} e^{z(x-\xi)}\left(\int_{0}^{\xi} e^{z t} \mathfrak{G}_{k}(\xi, t) d t\right) d \xi & =\int_{0}^{x}\left(\int_{0}^{\xi} e^{z(x+t-\xi)} \mathfrak{G}_{k}(\xi, t) d t\right) d \xi \\
& =\int_{0}^{x}\left(\int_{x-\xi}^{x} e^{z t} \mathfrak{G}_{k}(\xi, \xi+t-x) d t\right) d \xi \\
& =\int_{0}^{x} e^{z t}\left(\int_{x-t}^{x} \mathfrak{G}_{k}(\xi, \xi+t-x) d \xi\right) d t \\
& =\int_{0}^{x} e^{z t} \varkappa_{k+1}(x, t) d t \quad(k \geq 0) .
\end{aligned}
$$


Taking into account (2.25) and (2.27), we see that $g$ given by (2.21) satisfies the equation

$$
\frac{d}{d x} g(x, z)-z g(x, z)=\int_{0}^{x} e^{z t}\left(\sum_{k=0}^{\infty} \mathfrak{G}_{k}(x, t)\right) d t .
$$

In view of (2.26) we have the equalities

$$
\begin{aligned}
\int_{0}^{x} e^{z t} \varkappa(x, t) d t & =\int_{0}^{x} e^{z t} \mathfrak{G}_{0}(x, t) d t, \\
\int_{0}^{x} \varkappa(x, t) \int_{0}^{t} e^{z s} \varkappa_{k}(t, s) d s d t & =\int_{0}^{x} \varkappa(x, s) \int_{0}^{s} e^{z t} \varkappa_{k}(s, t) d t d s \\
& =\int_{0}^{x} e^{z t} \int_{t}^{x} \varkappa(x, s) \varkappa_{k}(s, t) d s d t \\
& =\int_{0}^{x} e^{z t} \mathfrak{G}_{k}(x, t) d t .
\end{aligned}
$$

Using (2.21), (2.25), (2.29), and (2.30), we rewrite (2.28) in the form (2.19).

It remains to prove that the solution of (2.19) is unique. Indeed, integrating (2.19) with respect to $x$ we derive the equality

$$
g(\cdot, z)-\operatorname{ARg}(\cdot, z)-z \operatorname{Ag}(\cdot, z)=I_{r},
$$

where the bounded in $L_{r}^{2}(0, \mathbf{T})$ operators $A$ and $R$ are given by the relations

$$
A f=\int_{0}^{x} f(t) d t, \quad R f=\int_{0}^{x} \varkappa(x, t) f(t) d t .
$$

Clearly $A$ is a Volterra operator and it is easily checked (see also, e.g., [17, Subsection 1.2.4] and [19]) that

$$
(I-z A)^{-1}=I+z \int_{0}^{x} e^{z(x-t)} \cdot d t .
$$

Therefore, $(I-z A)^{-1} A R$ is an integral triangular operator with Hilbert-Schmidt kernel (and so $(I-z A)^{-1} A R$ is also a Volterra operator). Hence, according to (2.31), the solution $g$ of (2.19) is uniquely defined by the formula

$$
g(\cdot, z)=\left(I-(I-z A)^{-1} A R\right)^{-1}(I-z A)^{-1} I_{r}
$$


Proof of Proposition 2.1. We split the proof into two steps. In the first step we construct the operator $E$ and establish the similarity $K E=E A$. In the next step we prove that $E^{ \pm 1}$ map functions with a square-summable derivative into functions with a square-summable derivative.

S TEP 1. Let $g(x, z)$ be the matrix function defined by (2.10). According to Lemma 2.2, $g(x, z)$ satisfies the equation (2.11). Hence, in view of Lemma 2.3, $g$ admits the representation

$$
g(x, z)=e^{z x} I_{r}+\int_{0}^{x} N(x, t)\left(e^{z t} I_{r}\right) d t, \quad 0 \leq x \leq \mathbf{T},
$$

where $N(x, t)$ is given by $(2.25)$. The same $N(x, t)$ is substituted into the definition (2.3) of the operator $E$ acting on $L_{r}^{2}(0, \mathbf{T})$, whereas the $r \times r$ matrix function $\rho$ in (2.3) coincides with $\rho$ defined by (2.9). Thus, the matrix functions $\rho, \rho^{-1}$ and $N$ are measurable and uniformly bounded, and $E$ is boundedly invertible.

Taking into account (2.3), (2.10), and (2.35) we see that

$$
E\left(e^{z x} I_{r}\right)=\rho(x) g(x, z)=(I-z K)^{-1} h,
$$

where $h$ is determined in (2.9) (i.e., $h(x)=F(x) G(0)$ ). It is immediate from (2.33) that

$$
e^{z x} I_{r}=(I-z A)^{-1} I_{r} .
$$

For the case that $z=0$ formula (2.36) yields $E I_{r}=h$. Thus, using (2.37), we rewrite (2.36) in the form

$$
E(I-z A)^{-1} I_{r}=(I-z K)^{-1} E I_{r} .
$$

From the series expansion in (2.38) it follows that

$$
E A^{j} I_{r}=K^{j} E I_{r}, \quad j=0,1,2, \ldots
$$

Therefore, for each $j=0,1,2, \ldots$, we have

$$
(K E) A^{j} I_{r}=K\left(E A^{j} I_{r}\right)=K^{j+1} E I_{r}=E A^{j+1} I_{r}=(E A) A^{j} I_{r} .
$$

As the closed linear span of the columns of the matrices $\left\{A^{j} I_{r}\right\}_{j=0}^{\infty}$ coincides with $L_{r}^{2}(0, \mathbf{T})$, the equalities in (2.40) yield $K E=E A$. Since $E$ is invertible, we obtain $K=E A E^{-1}$, and hence $K$ and $A$ are similar. It remains to prove that $E^{ \pm 1}$ map functions with a square-summable derivative into functions with a square-summable derivative. 
STEP 2. Let $f$ be a differentiable vector function such that

$$
\tilde{f}:=f^{\prime} \in L_{r}^{2}(0, \mathbf{T})
$$

Then $f$ admits a representation

$$
f=A \tilde{f}+f_{0} \quad\left(\tilde{f} \in L_{r}^{2}(0, \mathbf{T})\right), \quad f_{0} \equiv \text { const. }
$$

According to the previous step, $E I_{r}=h(x)=F(x) G(0)$, and so

$$
E f_{0}=F(x) G(0) f_{0}, \quad\left(E f_{0}\right)^{\prime}=F^{\prime}(x) G(0) f_{0} .
$$

Since we assume that the derivative $F^{\prime}$ is square-summable, the same is valid for $E f_{0}$. Next note that

$$
(E A \tilde{f})(x)=(K E \tilde{f})(x)=F(x) \int_{0}^{x} G(t)(E \tilde{f})(t) d t .
$$

Since $E$ maps $L_{r}^{2}(0, \mathbf{T})$ onto $L_{r}^{2}(0, \mathbf{T})$, formula (2.43) shows that $E A \tilde{f}$ has a square-summable derivative. Thus, both $E f_{0}$ and $E A \tilde{f}$ have square-summable derivatives. Therefore, (2.41) implies that $E f$ also has a square-summable derivative.

Finally, we consider $E^{-1}$. First, introduce operator $K_{1}$ on $L_{r}^{2}(0, \mathbf{T})$ :

$$
\left(K_{1} f\right)(x)=F^{\prime}(x) \int_{0}^{x} G(t) f(t) d t, \quad f \in L_{r}^{2}(0, \mathbf{T}),
$$

and notice that $A K_{1}=K-A$ or, equivalently,

$$
A\left(I+K_{1}\right)=K \text {. }
$$

The operator $K_{1}$ is a triangular operator with Hilbert-Schmidt kernel. In particular, $K_{1}$ is a Volterra operator. Thus, $I+K_{1}$ is invertible. Since $E$ is also invertible, we rewrite $K E=E A$ as $E^{-1} K=A E^{-1}$. In view of (2.44) the equality $E^{-1} K=A E^{-1}$ yields

$$
\begin{aligned}
E^{-1} A & =E^{-1} A\left(I+K_{1}\right)\left(I+K_{1}\right)^{-1} \\
& =E^{-1} K\left(I+K_{1}\right)^{-1} \\
& =A E^{-1}\left(I+K_{1}\right)^{-1} .
\end{aligned}
$$


Recall that $f$ with a square-summable derivative admits the representation of eq (2.41). Formula (2.45) implies that $E^{-1} A \tilde{f}$ has a square-summable derivative. In order to show that $E^{-1} f_{0}$ also has a square-summable derivative, we take into account (2.2) and rewrite the first equality in (2.42) in the form

$$
f_{0}=E^{-1}\left(F(x) G(0) f_{0}\right)=E^{-1} A\left(F^{\prime}(x) G(0) f_{0}\right)+E^{-1} f_{0},
$$

that is,

$$
E^{-1} f_{0}=f_{0}-E^{-1} A\left(F^{\prime}(x) G(0) f_{0}\right),
$$

which completes the proof.

Remark 2.4. Relations (2.41), (2.45), and (2.46) show that for any differentiable $f$ with a square-summable derivative we have

$$
\left(E^{-1} f\right)(0)=f(0)
$$

\section{Dirac system: fundamental solution}

We start with a similarity result, which follows from Proposition 2.1.

Proposition 3.1. Let the potential $v$ of Dirac system (1.1) be square-summable on $(0, \mathbf{T})$, and let $K$ be given by (1.11), where $\gamma$ is defined in (1.4). Then there is a similarity transformation operator $E \in B\left(L_{m_{2}}^{2}(0, \mathbf{T})\right)$ such that

$$
\begin{aligned}
& K=E A E^{-1}, \quad A:=-\mathrm{i} \int_{0}^{x} \cdot d t, \\
& E=I+\int_{0}^{x} N(x, t) \cdot d t, \\
& E^{-1} \gamma_{2} \equiv I_{m_{2}},
\end{aligned}
$$

where $N$ is a Hilbert-Schmidt kernel and $\gamma_{2}$ is the right $m_{2} \times m_{2}$ block of $\gamma$. Moreover, the operators $E^{ \pm 1}$ map differentiable functions with a square-summable derivative into differentiable functions with a square-summable derivative. 
Proof. According to (1.1) we have

$$
u(x, 0)^{*} j u(x, 0)=j=u(x, 0) j u(x, 0)^{*} .
$$

Therefore, the blocks of $u(x, 0)$ introduced in (1.4) satisfy the relations

$$
\beta j \beta^{*} \equiv I_{m_{1}}, \quad \gamma j \gamma^{*} \equiv-I_{m_{2}}, \quad \beta j \gamma^{*} \equiv 0
$$

Furthermore, equation (1.1) implies that $\gamma^{\prime}$ is square-summable and

$$
\gamma^{\prime}(x)=-\mathrm{i}\left[v(x)^{*} \quad 0\right] u(x, 0)=-\mathrm{i} v(x)^{*} \beta(x) .
$$

Hence, the third equality in (3.5) yields

$$
\gamma^{\prime} j \gamma^{*} \equiv 0
$$

In view of the second equality in (3.5), we may apply Proposition 2.1 to i $K$ (where $K$ is defined in (1.11)). Moreover, (3.6) implies the indentity $\rho(x) \equiv I_{r}$ for $\rho$ given in (2.3). Thus, there is some similarity transformation operator $\widetilde{E}$, which satisfies all conditions of Proposition 3.1 excluding, possibly, equality (3.3) (and the kernel of $\widetilde{E}$ is bounded). Let us normalize $\widetilde{E}$ multiplying it by the operator

$$
E_{0}=I+\int_{0}^{x} E_{0}(x-t) \cdot d t, \quad E_{0}(x):=\left(\widetilde{E}^{-1} \gamma_{2}\right)^{\prime}(x) .
$$

We see that $E=\widetilde{E} E_{0}$ admits representation (3.2), where $N$ is a Hilbert-Schmidt kernel and that $A E_{0}=E_{0} A$. Thus, from $K=\widetilde{E} A \widetilde{E}^{-1}$ follows $K=E A E^{-1}$. Finally, in view of (3.7) and Remark 2.4 we obtain

$$
\begin{aligned}
\left(E_{0} I_{m_{2}}\right)(x) & =I_{m_{2}}+\int_{0}^{x} E_{0}(t) d t \\
& =I_{m_{2}}+\left(\tilde{E}^{-1} \gamma_{2}\right)(x)-\left(\tilde{E}^{-1} \gamma_{2}\right)(0) \\
& =\left(\tilde{E}^{-1} \gamma_{2}\right)(x),
\end{aligned}
$$

and so (3.3) is valid for $E=\widetilde{E} E_{0}$.

Clearly, the equalities $A E_{0}=E_{0} A$ and (3.8) imply that $E_{0}$ maps differentiable functions with a square-summable derivative into differentiable functions with a square-summable derivative. Rewriting $A E_{0}=E_{0} A$ and (3.8) in the forms

$$
E_{0}^{-1} A=A E_{0}^{-1}, \quad E_{0}^{-1} I_{m_{2}}=I_{m_{2}}-\mathrm{i} E_{0}^{-1} A\left(\widetilde{E}^{-1} \gamma_{2}\right)^{\prime}=I_{m_{2}}-\mathrm{i} A E_{0}^{-1}\left(\widetilde{E}^{-1} \gamma_{2}\right)^{\prime},
$$

respectively, we see that $E_{0}^{-1}$ also maps differentiable functions with a squaresummable derivative into differentiable functions with a square-summable derivative. Thus, the same is valid for $E=\widetilde{E} E_{0}$ and for $E^{-1}$. 
Remark 3.2. Formulas $\widetilde{E}^{-1} A=A \widetilde{E}^{-1}$ and (2.46) for $\widetilde{E}^{-1}$ and formulas above for $E_{0}^{-1}$ yield a useful equality

$$
\left(E^{-1} \gamma_{1}\right)(0)=\left(E_{0}^{-1} \widetilde{E}^{-1} \gamma_{1}\right)(0)=\gamma_{1}(0)=0 .
$$

Now, we construct a representation of the fundamental solution $w$ of the system

$$
\frac{d}{d x} w(x, z)=\mathrm{i} z j \gamma(x)^{*} \gamma(x) w(x, z), \quad w(0, z)=I_{m} .
$$

For that purpose we introduce operators

$$
\begin{aligned}
& S:=E^{-1}\left(E^{*}\right)^{-1}, \quad \Pi:=\left[\begin{array}{ll}
\Phi_{1} & \Phi_{2}
\end{array}\right], \quad \Phi_{k} \in B\left(\mathbb{C}^{m_{k}}, L_{m_{2}}^{2}(0, l)\right) ; \\
& \left(\Phi_{1} f\right)(x)=\Phi_{1}(x) f, \quad \Phi_{1}(x):=\left(E^{-1} \gamma_{1}\right)(x) ; \quad \Phi_{2} f=I_{m_{2}} f \equiv f ;
\end{aligned}
$$

where $E$ is constructed (for the given $\gamma$ ) in Proposition 3.1 and $\gamma_{1}$ is the left $m_{2} \times m_{1}$ block of $\gamma$. We also introduce the transfer matrix function in Lev Sakhnovich form $[20,21,22]$

$$
w_{A}(z):=I_{m}+\mathrm{i} z j \Pi^{*} S^{-1}(I-z A)^{-1} \Pi .
$$

We shall need the reductions of the operators above (and the matrix function $w_{A}$ corresponding to those reductions):

$$
\begin{aligned}
& \left(P_{\xi} f\right)(x)=f(x) \quad(0<x<\xi), \quad P_{\xi} \in B\left(L_{m_{2}}^{2}(0, \mathbf{T}), L_{m_{2}}^{2}(0, \xi)\right), \\
& A_{\xi}:=P_{\xi} A P_{\xi}^{*}, \quad S_{\xi}:=P_{\xi} S P_{\xi}^{*}, \\
& w_{A}(\xi, z):=I_{m}+\mathrm{i} z j \Pi^{*} P_{\xi}^{*} S_{\xi}^{-1}\left(I-z A_{\xi}\right)^{-1} P_{\xi} \Pi, \quad 0<\xi \leq \mathbf{T} .
\end{aligned}
$$

Theorem 3.3. Let $\gamma$ be determined by (1.4), where $u$ is the fundamental solution of the Dirac system (1.1) with a square-summable potential $v$. Then, the fundamental solution $w$ given by (3.10) admits representation

$$
w(\xi, z)=w_{A}(\xi, z),
$$

where $w_{A}(\xi, z)$ is defined by (3.16).

Proof. Formulas (3.3), (3.11) and (3.12) imply that

$$
\Pi f=\left(E^{-1} \gamma\right) f \text {. }
$$

It is immediate from the definition (1.11) of $K$ that

$$
K^{*}=-\mathrm{i} \int_{x}^{\mathbf{T}} \gamma(x) j \gamma(t)^{*} \cdot d t, \quad K-K^{*}=\mathrm{i} \gamma(x) j \int_{0}^{\mathbf{T}} \gamma(t)^{*} \cdot d t .
$$


According to Proposition 3.1 we have $K=E A E^{-1}$. Since $K=E A E^{-1}$, taking into account (3.11) and (3.18), we rewrite the second equality in (3.19) in the form of the operator identity

$$
A S-S A^{*}=\mathrm{i} \Pi j \Pi^{*} .
$$

Hence, we may use the Method of Operator Identities [20, 21, 22]. We need now to show the applicability of the Continuous Factorization Theorem (see [22, p. 40]) or, more conveniently, its corollary [17, Theorem 1.20]. Completely similar to the cases in [17] we see that conditions $(i)$ and $(i i)$ of [17, Theorem 1.20] are satisfied. It remains only to derive that $\Pi^{*} P_{\xi} S_{\xi}^{-1} P_{\xi} \Pi$ is absolutely continuous (i.e., condition ( $i$ i i $)$ of [17, Theorem 1.20] holds) and that

$$
\left(\Pi^{*} P_{\xi}^{*} S_{\xi}^{-1} P_{\xi} \Pi\right)^{\prime}=H(\xi)=\gamma(\xi) \gamma(\xi)^{*},
$$

in order to prove that $w_{A}$ satisfies the differential system in (3.10).

Since the operator $E$ is invertible, triangular, and has Hilbert-Schmidt kernel, we see that $E^{-1}$ is also triangular. Taking into account that $E^{ \pm 1}$ are lower triangular operators, we obtain

$$
P_{\xi} E P_{\xi}^{*} P_{\xi}=P_{\xi} E, \quad\left(E^{-1}\right)^{*} P_{\xi}^{*}=P_{\xi}^{*} P_{\xi}\left(E^{-1}\right)^{*} P_{\xi}^{*} .
$$

The first equality in (3.22) yields $P_{\xi} E P_{\xi}^{*} P_{\xi} E^{-1} P_{\xi}^{*}=P_{\xi} P_{\xi}^{*}$, that is,

$$
P_{\xi} E^{-1} P_{\xi}^{*}=\left(P_{\xi} E P_{\xi}^{*}\right)^{-1} .
$$

Hence, formulas (3.11), (3.15), and (3.22) lead us to

$$
S_{\xi}^{-1}=E_{\xi}^{*} E_{\xi}, \quad E_{\xi}:=P_{\xi} E P_{\xi}^{*} .
$$

Finally, from (3.18), (3.22), and (3.23) we derive that

$$
\Pi^{*} P_{\xi}^{*} S_{\xi}^{-1} P_{\xi} \Pi=\int_{0}^{\xi} \gamma(\zeta) \gamma(\zeta)^{*} d \zeta
$$

(i.e., $\Pi^{*} P_{\xi}^{*} S_{\xi}^{-1} P_{\xi} \Pi$ is absolutely continuous and (3.21) is valid). Hence, $w_{A}$ satisfies the system in (3.10) and, furthermore, the normalization

$$
\lim _{x \rightarrow 0} w_{A}(x, z)=I_{m}
$$

easily follows from (3.16) and (3.23).

Since (3.20) holds we say that the triple $\{A, S, \Pi\}$ forms an $S$-node [20, 21, 22]. 
Corollary 3.4. Let $u(x, z)$ be the fundamental solution of a Dirac system with the square-summable potential $v$ and let $\gamma$ be given by (1.4). Then $u(x, z)$ admits representation

$$
u(x, z)=\mathrm{e}^{\mathrm{i} x z} u(x, 0) w_{A}(x, 2 z) .
$$

Here $w_{A}$ has the form (3.16), where the $S$-node $\{A, S, \Pi\}$, which determines $w_{A}$, is given in (3.1), (3.11), and (3.12).

Proof. According to (1.1) and Theorem 3.3 we have

$$
\begin{aligned}
& \left(\mathrm{e}^{\mathrm{i} x z} u(x, 0) w_{A}(x, 2 z)\right)^{\prime} \\
& \quad=\left(\mathrm{i} z I_{m}+\mathrm{i} j V(x)+2 \mathrm{i} z u(x, 0) j \gamma(x)^{*} \gamma(x) u(x, 0)^{-1}\right) \mathrm{e}^{\mathrm{i} x z} u(x, 0) w_{A}(x, 2 z) .
\end{aligned}
$$

Writing $u(x, 0)$ in the block form and taking into account (3.5), we derive

$$
u(x, 0)=\left[\begin{array}{l}
\beta(x) \\
\gamma(x)
\end{array}\right], \quad u(x, 0) j \gamma(x)^{*}=\left[\begin{array}{c}
0 \\
-I_{m_{2}}
\end{array}\right] .
$$

From (3.4) we obtain $u(x, 0)^{-1}=j u(x, 0)^{*} j$. Thus, in view of (3.27) and (3.28) we see that

$$
\begin{aligned}
& \left(\mathrm{e}^{\mathrm{i} x z} u(x, 0) w_{A}(x, 2 z)\right)^{\prime} \\
& \quad=\left(\mathrm{i} z I_{m}+\mathrm{i} j V(x)-2 \mathrm{i} z\left[\begin{array}{cc}
0 & 0 \\
0 & I_{m_{2}}
\end{array}\right]\right) \mathrm{e}^{\mathrm{i} x z} u(x, 0) w_{A}(x, 2 z) \\
& \quad=(\mathrm{i} z j+\mathrm{i} j V(x)) \mathrm{e}^{\mathrm{i} x z} u(x, 0) w_{A}(x, 2 z) .
\end{aligned}
$$

Relations (3.25) and (3.29) yield (3.26).

\section{Solution of the inverse problem}

Here, we may follow the lines of [5, Sections 3 and 4] without any essential changes. The high-energy asymptotics of $\varphi$ is given by the following theorem.

Theorem 4.1. Assume that $\varphi \in \mathcal{N}(\mathbf{T}, z)$ and the potential $v$ of the corresponding Dirac system (1.1) is square-summable on $(0, \mathbf{T})$. Then (uniformly with respect to $\Re(z)$ ) we have

$$
\varphi(z)=2 \mathrm{i} z \int_{0}^{\mathbf{T}} \mathrm{e}^{2 \mathrm{i} x z} \Phi_{1}(x) d x+O\left(2 z \mathrm{e}^{2 \mathrm{i} \mathbf{T} z} / \sqrt{\Im(z)}\right), \quad \Im(z) \rightarrow \infty .
$$


Proof. To prove the theorem, we consider the matrix function

$$
\mathcal{U}(z)=\left[\begin{array}{ll}
I_{m_{1}} & \varphi(z)^{*}
\end{array}\right]\left(j-w_{A}(\mathbf{T}, 2 z)^{*} j w_{A}(\mathbf{T}, 2 z)\right)\left[\begin{array}{c}
I_{m_{1}} \\
\varphi(z)
\end{array}\right] .
$$

It easily follows from (3.16) and (3.20) (see, e.g., [17, p. 24]) that

$$
w_{A}(\mathbf{T}, z)^{*} j w_{A}(\mathbf{T}, z)=j+\mathrm{i}(z-\bar{z}) \Pi^{*}\left(I-\bar{z} A^{*}\right)^{-1} S^{-1}(I-z A)^{-1} \Pi,
$$

and so we derive $\mathcal{U}(z) \geq 0$. Because of (3.4), (3.26), and (4.2) we have

$$
u(z)=I_{m_{1}}-\varphi(z)^{*} \varphi(z)-\mathrm{e}^{\mathrm{i} \mathbf{T}(\bar{z}-z)}\left[I_{m_{1}} \varphi(z)^{*}\right] u(\mathbf{T}, z)^{*} j u(\mathbf{T}, z)\left[\begin{array}{c}
I_{m_{1}} \\
\varphi(z)
\end{array}\right] .
$$

We note that (1.8) yields

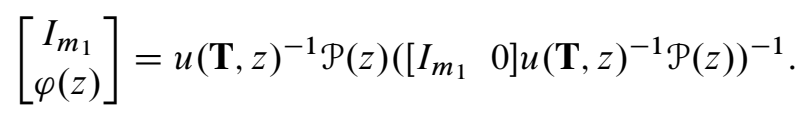

Taking into account (4.5), we rewrite (4.4) as

$$
\begin{aligned}
\mathcal{U}(z)= & I_{m_{1}}-\varphi(z)^{*} \varphi(z)-\mathrm{e}^{\mathrm{i} \mathbf{T}(\bar{z}-z)}\left(\left(\left[I_{m_{1}} 0\right] u(\mathbf{T}, z)^{-1} \mathcal{P}(z)\right)^{-1}\right)^{*} \\
& \times \mathcal{P}(z)^{*} j \mathcal{P}(z)\left(\left[\begin{array}{ll}
I_{m_{1}} & 0
\end{array}\right] u(\mathbf{T}, z)^{-1} \mathcal{P}(z)\right)^{-1} .
\end{aligned}
$$

Recall that $\mathcal{U}(z) \geq 0$. Hence, from (1.6) and (4.6) we see that

$$
0 \leq \mathcal{U}(z) \leq I_{m_{1}}, \quad \varphi(z)^{*} \varphi(z) \leq I_{m_{1}} .
$$

Now, formulas (4.2), (4.3), and (4.7) imply that

$$
2 \mathrm{i}(\bar{z}-z)\left[\begin{array}{ll}
I_{m_{1}} & \varphi(z)^{*}
\end{array}\right] \Pi^{*}\left(I-2 \bar{z} A^{*}\right)^{-1} S^{-1}(I-2 z A)^{-1} \Pi\left[\begin{array}{c}
I_{m_{1}} \\
\varphi(z)
\end{array}\right] \leq I_{m_{1}} .
$$

Since $S$ is positive and boundedly invertible, inequality (4.8) yields

$$
\left\|(I-2 z A)^{-1} \Pi\left[\begin{array}{c}
I_{m_{1}} \\
\varphi(z)
\end{array}\right]\right\| \leq C / \sqrt{\Im z} \text { for some } C>0 .
$$

After applying $-\mathrm{i} \Phi_{2}^{*}$ to the operator on the left-hand side of (4.9), we derive

$$
-\mathrm{i} \Phi_{2}^{*}(I-2 z A)^{-1} \Phi_{2} \varphi(z)=\mathrm{i} \Phi_{2}^{*}(I-2 z A)^{-1} \Phi_{1}+O\left(\frac{1}{\sqrt{\Im}(z)}\right) .
$$

Using (2.33) we see that

$$
\begin{aligned}
& \Phi_{2}^{*}(I-2 z A)^{-1} f=\int_{0}^{\mathbf{T}} \mathrm{e}^{2 \mathrm{i}(x-\mathbf{T}) z} f(x) d x, \\
& \Phi_{2}^{*}(I-2 z A)^{-1} \Phi_{2}=\frac{\mathrm{i}}{2 z}\left(\mathrm{e}^{-2 \mathrm{i} \mathbf{T} z}-1\right) I_{m_{2}} .
\end{aligned}
$$


Because of (4.10)-(4.12), we have

$$
\frac{1}{2 z}\left(\mathrm{e}^{-2 \mathrm{i} \mathbf{T} z}-1\right) \varphi(z)=\mathrm{ie}^{-2 \mathrm{i} \mathbf{T} z} \int_{0}^{\mathbf{T}} \mathrm{e}^{2 \mathrm{i} x z} \Phi_{1}(x) d x+O\left(\frac{1}{\sqrt{\Im(z)}}\right) .
$$

Since $\varphi$ is non-expansive, we see from (4.13) that (4.1) holds.

Corollary 4.2. Let $\varphi$ be the Weyl function of Dirac system $(1.1)$ on $[0, \infty)$, where the potential $v$ is locally square-summable. Then we have

$$
\varphi(z)=2 \mathrm{i} z \int_{0}^{\infty} \mathrm{e}^{2 \mathrm{i} x z} \Phi_{1}(x) d x, \quad \Im(z)>0 .
$$

Proof. Since $\varphi$ is analytic and non-expansive in $\mathbb{C}_{+}$, for any $\varepsilon>0$ it admits (see, e.g., [14, Theorem V]) a representation

$$
\varphi(z)=2 \mathrm{i} z \int_{0}^{\infty} \mathrm{e}^{2 \mathrm{i} x z} \Phi(x) d x, \quad \Im(z)>\varepsilon>0,
$$

where $\mathrm{e}^{-2 \varepsilon x} \Phi(x) \in L_{m_{2} \times m_{1}}^{2}(0, \infty)$. Because of (4.1) and (4.15) we obtain

$$
\begin{aligned}
\psi(z): & =\int_{0}^{\mathbf{T}} \mathrm{e}^{2 \mathrm{i}(x-\mathbf{T}) z}\left(\Phi_{1}(x)-\Phi(x)\right) d x \\
& =\int_{\mathbf{T}}^{\infty} \mathrm{e}^{2 \mathrm{i}(x-\mathbf{T}) z} \Phi(x) d x+O\left(\frac{1}{\sqrt{\Im(z)}}\right) .
\end{aligned}
$$

From (4.16) we see that $\psi(z)$ is bounded in some half-plane $\Im(z) \geq \eta_{0}>0$. Clearly, $\psi(z)$ is bounded also in the half-plane $\Im(z)<\eta_{0}$. Since $\psi$ is analytic and bounded in $\mathbb{C}$ and tends to zero on some rays, we have

$$
\psi(z)=\int_{0}^{\mathbf{T}} \mathrm{e}^{2 \mathrm{i}(x-\mathbf{T}) z}\left(\Phi_{1}(x)-\Phi(x)\right) d x \equiv 0 .
$$

It follows from (4.17) that $\Phi_{1}(x) \equiv \Phi(x)$ on all finite intervals [0, T]. Hence, (4.15) implies (4.14).

Remark 4.3. According to the proof of Corollary 4.2, we have $\Phi_{1} \equiv \Phi$, and so $\Phi_{1}(x)$ does not depend on $\mathbf{T}$ for $\mathbf{T}>x$. Furthermore, the proof of Corollary 4.2 implies also that $\mathrm{e}^{-\varepsilon x} \Phi_{1}(x) \in L_{m_{2} \times m_{1}}^{2}(0, \infty)$ for any $\varepsilon>0$. 
Using representation (4.14), we uniquely recover $v$ from $\varphi$. Indeed, taking into account Plancherel Theorem and Remark 4.3, we apply inverse Fourier transform to formula (4.14) and derive

$$
\Phi_{1}\left(\frac{x}{2}\right)=\frac{1}{\pi} \mathrm{e}^{x \eta} 1 . \mathrm{i} \cdot \mathrm{m}_{\cdot a \rightarrow \infty} \int_{-a}^{a} \mathrm{e}^{-\mathrm{i} x \xi} \frac{\varphi(\xi+\mathrm{i} \eta)}{2 \mathrm{i}(\xi+\mathrm{i} \eta)} d \xi, \quad \eta>0 .
$$

Here 1.i.m. stands for the entrywise limit in the norm of $L^{2}(0, b), 0<b \leq \infty$. (Note that if we put additionally $\Phi_{1}(x)=0$ for $x<0$, equality (4.18) holds for l.i.m. as the entrywise limit in $L^{2}(-b, b)$.) Thus, for any fixed interval $(0, \mathbf{T})$ the corresponding operators $S$ and $\Pi$ are recovered from $\varphi$.

Since the Hamiltonian $H$ is recovered from $S$ and $\Pi$ via formula (3.21), and $H=\gamma^{*} \gamma$, we recover also $\gamma$. First, for that purpose, we recover the so called Schur coefficient:

$$
\left(\left[\begin{array}{ll}
0 & I_{m_{2}}
\end{array}\right] H\left[\begin{array}{c}
0 \\
I_{m_{2}}
\end{array}\right]\right)^{-1}\left[\begin{array}{ll}
0 & I_{m_{2}}
\end{array}\right] H\left[\begin{array}{c}
I_{m_{1}} \\
0
\end{array}\right]=\left(\gamma_{2}^{*} \gamma_{2}\right)^{-1} \gamma_{2}^{*} \gamma_{1}=\gamma_{2}^{-1} \gamma_{1} .
$$

Here we used the inequality $\operatorname{det} \gamma_{2} \neq 0$, which follows from the second identity in (3.5). The second identity in (3.5) yields also

$$
I_{m_{2}}-\left(\gamma_{2}^{-1} \gamma_{1}\right)\left(\gamma_{2}^{-1} \gamma_{1}\right)^{*}=\gamma_{2}^{-1}\left(\gamma_{2}^{-1}\right)^{*},
$$

which implies that the left-hand side of this equality is invertible. Taking into account det $\gamma_{2} \neq 0$, we rewrite $\gamma_{1}$ in the form $\gamma_{1}=\gamma_{2}\left(\gamma_{2}^{-1} \gamma_{1}\right)$ and the identity (3.6) in the form $\gamma_{2}^{\prime}=\gamma_{1}^{\prime}\left(\gamma_{2}^{-1} \gamma_{1}\right)^{*}$. Therefore, we obtain

$$
\gamma_{2}^{\prime}=\left(\gamma_{2}\left(\gamma_{2}^{-1} \gamma_{1}\right)\right)^{\prime}\left(\gamma_{2}^{-1} \gamma_{1}\right)^{*}
$$

i.e.

$$
\gamma_{2}^{\prime}=\gamma_{2}\left(\gamma_{2}^{-1} \gamma_{1}\right)^{\prime}\left(\gamma_{2}^{-1} \gamma_{1}\right)^{*}\left(I_{m_{2}}-\left(\gamma_{2}^{-1} \gamma_{1}\right)\left(\gamma_{2}^{-1} \gamma_{1}\right)^{*}\right)^{-1},
$$

and recover $\gamma_{2}$ from (4.20) and the initial condition $\gamma_{2}(0)=I_{m_{2}}$. Finally, we recover $\gamma_{1}$ from $\gamma_{2}$ and $\gamma_{2}^{-1} \gamma_{1}$.

In order to recover $\beta$ from $\gamma$, we partition $\beta$ into two blocks $\beta=\left[\begin{array}{ll}\beta_{1} & \beta_{2}\end{array}\right]$, where $\beta_{k}(k=1,2)$ is an $m_{1} \times m_{k}$ matrix function. We put

$$
\tilde{\beta}=\left[\begin{array}{ll}
I_{m_{1}} & \gamma_{1}^{*}\left(\gamma_{2}^{*}\right)^{-1}
\end{array}\right] .
$$

Because of (3.5) and (4.21), we have $\beta j \gamma^{*}=\tilde{\beta} j \gamma^{*}=0$, and so

$$
\beta(x)=\beta_{1}(x) \tilde{\beta}(x) .
$$


It follows from (1.1) and (1.4) that

$$
\beta^{\prime}(x)=\mathrm{i} v(x) \gamma(x),
$$

which implies

$$
\beta^{\prime} j \beta^{*}=0, \quad \beta^{\prime} j \gamma^{*}=-\mathrm{i} v .
$$

Formula (4.22) and the first relation in (3.5) lead us to

$$
\tilde{\beta} j \tilde{\beta}^{*}=\beta_{1}^{-1}\left(\beta_{1}^{*}\right)^{-1} .
$$

From (4.22) we also derive that

$$
\beta^{\prime} j \beta^{*}=\beta_{1}^{\prime}\left(\tilde{\beta} j \tilde{\beta}^{*}\right) \beta_{1}^{*}+\beta_{1}\left(\tilde{\beta}^{\prime} j \tilde{\beta}^{*}\right) \beta_{1}^{*} .
$$

Taking into account the first relation in (4.24) and formula (4.25), we rewrite the relation above:

$$
\beta_{1}^{\prime} \beta_{1}^{-1}+\beta_{1}\left(\tilde{\beta}^{\prime} j \tilde{\beta}^{*}\right) \beta_{1}^{*}=0 .
$$

According to (1.3), (4.25), and (4.26), $\beta_{1}$ satisfies the first order differential equation (and initial condition):

$$
\beta_{1}^{\prime}=-\beta_{1}\left(\tilde{\beta}^{\prime} j \tilde{\beta}^{*}\right)\left(\tilde{\beta} j \tilde{\beta}^{*}\right)^{-1}, \quad \beta_{1}(0)=I_{m_{1}} .
$$

Thus, $\beta_{1}$ and $\beta$ are successively recovered from $\gamma$. The potential $v$ is recovered from $\beta$ and $\gamma$ via the second equality in (4.24). In this way, we recover $v$ on any interval $[0, \mathbf{T}]$, therefore, on the whole semiaxis. We proved the following theorem.

Theorem 4.4. Let $\varphi$ be the Weyl function of Dirac system (1.1) on $[0, \infty)$, where the potential $v$ is locally square-summable. Then $v$ can be uniquely recovered from $\varphi$ via the formula

$$
v(x)=\mathrm{i} \beta^{\prime}(x) j \gamma(x)^{*} .
$$

Here $\beta$ is recovered from $\gamma$ using (4.21), (4.22) and (4.27); $\gamma$ is recovered from the Hamiltonian $H$ using (4.19) and (4.20); the Hamiltonian is given by (3.21), $\Pi$ from (3.21) is expressed via $\Phi_{1}(x)$ in formula (3.12), and $S$ is the unique solution of (3.20). Finally, $\Phi_{1}(x)$ is recovered from $\varphi$ using (4.18). 
Remark 4.5. It follows from (3.15) and (3.20) that the operator identities

$$
A_{\xi} S_{\xi}-S_{\xi} A_{\xi}^{*}=\mathrm{i} P_{\xi} \Pi j\left(P_{\xi} \Pi\right)^{*}, \quad 0<\xi \leq \mathbf{T},
$$

where $A$ is given in (3.1), $A_{\xi}=P_{\xi} A P_{\xi}^{*}$, and $\Pi$ is given by (3.12), hold. The uniqueness of the operators $S_{\xi}$ satisfying these identities is proved on p. 311 in [17]. Moreover, it is easy to see that the proof of [7, Proposition 3.2] works also for the case where $\psi$ and $\tilde{\psi}$ are differentiable functions with the square-summable derivatives. Thus, recalling (3.9) and formulas (3.16) and (3.17) in [7, Proposition 3.2], we see that $S_{\xi}$ given by

$$
S_{\xi}=I-\frac{1}{2} \int_{0}^{\xi} \int_{|x-t|}^{x+t} \Phi_{1}^{\prime}\left(\frac{\zeta+x-t}{2}\right) \Phi_{1}^{\prime}\left(\frac{\zeta+t-x}{2}\right)^{*} d \zeta \cdot d t
$$

satisfies (4.29). Hence, $S_{\xi}$ of the form (4.30) is the unique solution of (4.29), and we may recover $S_{\xi}$ (considered in Theorem 4.4) from $\Phi_{1}$ in this way.

Using Theorem 4.4 we modify Borg-Marchenko-type Theorem 2.52 from [17] for the case of the locally square-summable potentials. We note that seminal publications by F. Gesztesy and B. Simon [9, 10, 23] gave rise to a series of interesting results on the high energy asymptotics of the Weyl functions and local BorgMarchenko-type uniqueness theorems. Recall that the high energy asymptotics of the Weyl functions is given (for our case) in Theorem 4.1.

Theorem 4.6. Let $\varphi$ and $\hat{\varphi}$ be Weyl functions of two Dirac systems on $[0, \mathbf{T}]$ (or on $[0, \infty)$ ) with square-summable (locally square-summable) potentials, which are denoted by $v$ and $\hat{v}$, respectively. Suppose that on some ray $\Re z=c \mathfrak{s} z$, where $c \in \mathbb{R}$ and $\Im z>0$, the equality

$$
\|\varphi(z)-\hat{\varphi}(z)\|=O\left(\mathrm{e}^{2 \mathrm{i} \zeta z}\right) \quad(\mathfrak{\Im} z \rightarrow \infty)
$$

holds for all $0<\zeta<l(l<\mathbf{T}<\infty)$. Then we have

$$
v(x)=\hat{v}(x), \quad 0<x<l .
$$

Proof. Since Weyl functions are non-expansive, it is immediate that the inequality

$$
\left\|\mathrm{e}^{-2 \mathrm{i} \zeta z}(\varphi(z)-\hat{\varphi}(z))\right\| \leq c_{1} \mathrm{e}^{2 \zeta|z|}, \quad \Im z \geq c_{2}>0
$$

is valid for some $c_{1}$ and $c_{2}$. It is apparent also that the matrix function $\mathrm{e}^{-2 \mathrm{i} \zeta z}(\varphi(z)-\hat{\varphi}(z))$ is bounded on the line $\Im z=c_{2}$. Furthermore, formula (4.31) implies that $\mathrm{e}^{-2 \mathrm{i} \xi z}(\varphi(z)-\hat{\varphi}(z))$ is bounded on the ray $\Re z=c \mathfrak{I} z$. Therefore, 
applying the Phragmen-Lindelöf theorem (e.g. its version [17, Corollary E.7]) in the angles generated by the line $\Im z=c_{2}$ and the ray $\Re z=c \Im z\left(\Im z \geq c_{2}\right)$, we see that

$$
\left\|\mathrm{e}^{-2 i \zeta z}(\varphi(z)-\hat{\varphi}(z))\right\| \leq c_{3}, \quad \Im z \geq c_{2}>0 .
$$

Let functions associated with $\hat{\varphi}$ be written with a hat (e.g., $\hat{v}, \widehat{\Phi}_{1}$ ). Because of formula (4.1), its analog for $\hat{\varphi}, \widehat{\Phi}_{1}$ and the inequality (4.34), we have

$$
\left\|\int_{0}^{\zeta} \mathrm{e}^{2 \mathrm{i}(x-\zeta) z}\left(\Phi_{1}(x)-\widehat{\Phi}_{1}(x)\right) d x\right\| \leq c_{4}, \quad \Im z \geq c_{2}>0 .
$$

Clearly, the left-hand side of (4.35) is bounded in the half-plane $\Im z<c_{2}$ and tends to zero on some rays. Thus, we derive

$$
\int_{0}^{\zeta} \mathrm{e}^{2 \mathrm{i}(x-\zeta) z}\left(\Phi_{1}(x)-\widehat{\Phi}_{1}(x)\right) d x \equiv 0,
$$

i.e.

$$
\Phi_{1}(x) \equiv \widehat{\Phi}_{1}(x) \quad(0<x<\zeta) .
$$

Since (4.36) holds for all $\zeta<l$, we obtain $\Phi_{1}(x) \equiv \widehat{\Phi}_{1}(x)$ for $0<x<l$. In view of Theorem 4.4, the last identity implies (4.32).

Acknowledgement. The research was supported by the Austrian Science Fund (FWF) under Grant No. P24301. The author is grateful to F. Gesztesy for his question, which initiated this paper.

\section{References}

[1] S. Albeverio, R. Hryniv, and Ya. Mykytyuk, Reconstruction of radial Dirac operators. J. Math. Phys. 48 (2007), no. 4, article id. 043501. MR 2319895 Zbl 1132.47060

[2] D. Alpay, I. Gohberg, M. A. Kaashoek, L. Lerer, and A. Sakhnovich, Krein systems and canonical systems on a finite interval: accelerants with a jump discontinuity at the origin and continuous potentials. Integral Equations Operator Theory 68 (2010), no. 1115-150. MR 2677891 Zbl 1206.34025

[3] S. Clark and F. Gesztesy, On self-adjoint and $J$-self-adjoint Dirac-type operators: a case study. In N. Chernov, Y. Karpeshina, I. W. Knowles, R. T. Lewis and R. Weikard (eds.), Recent advances in differential equations and mathematical physics. Papers from the $10^{\text {th }}$ International Conference on Differential Equations and Mathematical Physics held at the University of Alabama at Birmingham, Birmingham, AL, March 29-April 2, 2005. Contemporary Mathematics, 412. American Mathematical Society, Providence, R.I., 2006, 103-140. MR 2259103 MR 2259040 (collection) Zbl 1124.34062 Zbl 1097.34002 (collection) 
[4] J. Eckhardt, F. Gesztesy, R. Nichols, and G. Teschl, Supersymmetry and Schrödinger-type operators with distributional matrix-valued potentials. J. Spectr. Theory 4 (2014), no. 4, 715-768. MR 3299812 Zbl 06424789

[5] B. Fritzsche, B. Kirstein, I. Ya. Roitberg, and A. L. Sakhnovich, Recovery of the Dirac system from the rectangular Weyl matrix function. Inverse Problems 28 (2012), no. 1, article id. 015010. MR 2864505 Zbl 1242.34027

[6] B. Fritzsche, B. Kirstein, I. Ya. Roitberg, and A. L. Sakhnovich, Weyl theory and explicit solutions of direct and inverse problems for Dirac system with a rectangular matrix potential. Oper. Matrices 7 (2013), no. 1, 183-196. MR 3076466 Zbl 1281.34030

[7] B. Fritzsche, B. Kirstein, and A. L. Sakhnovich, Semiseparable integral operators and explicit solution of an inverse problem for the skew-self-adjoint Dirac-type system. Integral Equations Operator Theory 66 (2010), 231-251. MR 2595655 Zbl 1198.34019

[8] F. Gesztesy, J. A. Goldstein, H. Holden, and G. Teschl, Abstract wave equations and associated Dirac-type operators. Ann. Mat. Pura Appl. (4) 191, no. 4 (2012), 631-676. MR 2993967 Zbl 1262.35174

[9] F. Gesztesy and B. Simon, On local Borg-Marchenko uniqueness results. Commun. Math. Phys. 211 (2000), no. 2, 273-287. MR 1754515 Zbl 0985.34077

[10] F. Gesztesy and B. Simon, A new approach to inverse spectral theory. II. General real potentials and the connection to the spectral measure. Ann. of Math. (2) 152 (2000), 593-643. MR 1804532 Zbl 0983.34013

[11] M. G. Krein, Continuous analogues of propositions on polynomials orthogonal on the unit circle. Dokl. Akad. Nauk SSSR 105 (1955), 637-640. MR 0080735

[12] Ya. V. Mykytyuk and D. V. Puyda, Inverse spectral problems for Dirac operators on a finite interval. J. Math. Anal. Appl. 386 (2012), no. 1, 177-194. MR 2834876 Zbl 1264.34025

[13] D. V. Puyda, Inverse spectral problems for Dirac operators with summable matrixvalued potentials. Integral Equations Operator Theory 74 (2012), no. 3, 417-450. MR 2992031 Zbl 1268.34042

[14] R. E. A. C. Paley and N. Wiener, Fourier transforms in the complex domain. Reprint of the 1934 original. American Mathematical Society Colloquium Publications, 19. American Mathematical Society, Providence, R.I., 1987. MR 1451142 Zbl 0011.01601

[15] A. L. Sakhnovich, Asymptotic behavior of spectral functions of an $S$-node. Izv. Vyssh. Uchebn. Zaved. Mat. 1988, no. 9, 62-72. In Russian. English transl., Soviet Math. (Iz. VUZ) 32 (1988), no. 9, 92-105. MR 0982593 Zbl 0674.47017

[16] A. L. Sakhnovich, Dirac type and canonical systems: spectral and Weyl-Titchmarsh functions, direct and inverse problems. Inverse Problems 18 (2002), no. 2, 331-348. MR 1910241 Zbl 1009.34079

[17] A. L. Sakhnovich, L. A. Sakhnovich, and I. Ya. Roitberg, Inverse problems and nonlinear evolution equations. Solutions, Darboux matrices and Weyl-Titchmarsh functions. De Gruyter Studies in Mathematics, 47. De Gruyter, Berlin, 2013. MR 3098432 Zbl 1283.47003 
[18] L. A. Sakhnovich, Spectral analysis of Volterra's operators defined in the space of vector-functions $L_{m}^{2}(0, l)$. Ukrain. Mat. Ž. 16 (1964), no. 2 259-268. In Russian. English transl. Amer. Math. Soc. Transl. (2) 61 (1967), 85-95. MR 0165396 Zbl 0192.23103

[19] L. A. Sakhnovich, The operator Bezoutiant in the theory of the separation of roots of entire functions. Funkcional. Anal. i Prilozhen. 10 (1976), no 1, 54-61. MR 0430842

[20] L. A. Sakhnovich, On the factorization of an operator-valued transfer function. Dokl. Akad. Nauk SSSR 226 (1976), no. 4, 781-784. In Russian. English transl., Sov. Math. Dokl. 17 (1976), 203-207. MR 0407655 Zbl 0342.93022

[21] L. A. Sakhnovich, Integral equations with difference kernels on finite intervals. Translated from the Russian manuscript by E. Melnichenko. Operator Theory: Advances and Applications, 84. Birkhäuser Verlag, Basel, 1996. MR 1383591 Zbl 0841.45003

[22] L. A. Sakhnovich, Spectral theory of canonical differential systems. Method of operator identities. Translated from the Russian manuscript by E. Melnichenko. Operator Theory: Advances and Applications, 107. Birkhäuser Verlag, Basel, 1999. MR 1690379 Zbl 0918.47003

[23] B. Simon, A new approach to inverse spectral theory. I. Fundamental formalism. Ann. of Math. (2) 150 (1999), 1029-1057. MR 1740987 Zbl 0945.34013

Received January 5, 2014

Alexander Sakhnovich, Address: Fakultät für Mathematik, Universität Wien,

Oskar-Morgenstern-Platz 1, A-1090 Wien, Austria

e-mail: oleksandr.sakhnovych@univie.ac.at 